\title{
Early Diagnosis of Necrotizing Enterocolitis in Neonates Using an Electronic Nose Mira Prabhakar ${ }^{1}$, Troy Markel ${ }^{1,2}$ \\ ${ }^{1}$ Indiana University School of Medicine; ${ }^{2}$ Pediatric Surgery Department at Riley at IU Health
}

\begin{abstract}
Background: Necrotizing enterocolitis (NEC) is a devastating clinical problem that often necessitates resection of necrotic intestine, leaving infants with a suboptimal length of intestine to absorb nutrition. There are no adequate tests or biomarkers to predict NEC onset. We hypothesized that assessment of biological samples with a novel electronic nose can be used with machine learning algorithms to detect aberrancies in stool and urine volatile organic compounds to predict NEC risk.
\end{abstract}

Methods: 18 infants of gestational age $\leq 34$ weeks in the Riley Hospital NICU were enrolled in the study and underwent stool (38 control, $3 \mathrm{NEC)}$ and urine (42 control, $5 \mathrm{NEC}$ ) sample collection. Stool was collected by the bedside nurse via the infant's diaper or ostomy bag. Urine was collected using a bag around the infant's perineum or via foley catheter if already present. Stool samples and 250uL urine samples were aliquoted into Eppendorf tubes and covered with Parafilm. Samples were heated to $40^{\circ} \mathrm{C}, 30$ minutes for stool, and 10 minutes for urine. Sample headspace was analyzed using the Cyranose 320 electronic nose, creating a "smellprint" comprised of readings from 32 unique sensors. Individual sensors were compared using MannWhitney $U$ test. $P<0.05$ was significant.

Results: There was no significant difference in urine or stool signals among the 32 sensors between NEC and control groups.

Potential Impact: While no significant difference was found, this study is greatly limited by the number of patients enrolled, with only 2 of the 18 being diagnosed with NEC. Past retrospective studies with this device have found differences between NEC and non-NEC stool. The future goals of this study are to continue enrolling patients to have a more robust data set. Using machine learning, we aim to create a model to predict NEC before its clinical manifestation so that beneficial treatment can be initiated earlier.

Acknowledgment: This project was funded, in part, with support from the NIH NHLBI ShortTerm Training Program in Biomedical Sciences Grant funded, in part by T35HL110854 from the National Institutes of Health. The content is solely the responsibility of the authors and does not necessarily represent the official views of the National Institutes of Health. 